\title{
MiR-491-3p is down-regulated in postmenopausal osteoporosis and affects growth, differentiation and apoptosis of hFOB1.19 cells through targeting CTSS
}

\author{
Wen-Xiong Hu, Hua Li, Jia-Zheng Jiang \\ Department of Orthopedics, Hainan Western Central Hospital, Hainan Province, P.R. China
}

\begin{abstract}
Background. Postmenopausal osteoporosis (PMO) is a common disease related to aging, which has been paid increasing attention in recent years because of its serious complications. MiR-491-3p was found to play a crucial roles in several diseases. However, the role of miR-491-3p in PMO has yet not been studied. Our research intends to explore the impact of miR-491-3p on PMO in the in vitro model.

Material and methods. The expression patterns of miR-491-3p and cathepsin S (CTSS) in patients with PMO were acquired from the GEO database. The human osteoblast cell hFOB1.19 was used to detect the function of miR-491-3p and CTSS in PMO. The viability and apoptosis of hFOB1.19 cells were measured by cell counting kit 8 and flow cytometry assays. The apoptosis and differentiation related proteins were analyzed by western blotting. The relationship between miR-491-3p and CTSS was predicted by appropriate software and affirmed by luciferase assay.

Results. MiR-491-3p expression was lower in patients with PMO. The up-regulation of miR-491-3p in hFOB1.19 cells increased their viability and differentiation and inhibited their apoptosis. CTSS, which was highly expressed in patients with PMO, was confirmed as a direct target of miR-491-3p and was found to be inversely modulated by miR-491-3p. The rescue assays showed that overexpression of CTSS suppressed the promoting effects of miR491-3p mimic on the proliferation and differentiation of hFOB1.19 cells, and repressed the inhibitory effects of miR-491-3p mimic on apoptosis of hFOB1.19 cells.

Conclusions. The results of our study showed that miR-491-3p could ameliorate biological characteristics of hFOB1.19 cells by reducing CTSS expression suggesting that miR-491-3p/CTSS might be a potential biomarker for the diagnosis and treatment of PMO. (Folia Histochemica et Cytobiologica 2020, Vol. 58, No. 1, 9-16)
\end{abstract}

Key words: postmenopausal osteoporosis; osteoblastic hFOB1.19 cells; miR-491-3p; cathepsin S; proliferation; apoptosis; flow cytometry

\section{Introduction}

In recent years, an increasing number of women over the age of 50 are being affected by the postmenopausal osteoporosis (PMO), which leads to an increased economic and social burden [1]. Bone health is mainly dependent on the dynamic balance between bone formation and bone resorption. Disorders of bone metabolism easily induce bone-related diseases, especial-

Correspondence address: Wen-Xiong $\mathrm{Hu}$

Department of Orthopedics,

Hainan Western Central Hospital,

Hainan Province, P.R. China

e-mail: Hnhwx2010@sina.cn ly osteoporosis, which can result in a decrease in bone density and an increase in the risk of fracture $[2,3]$. Bone homeostasis is a remolding procedure regulated by three types of osteocytes, including osteoclast, osteoblast, and osteocyte [4]. PMO is considered to be a direct result of the decrease of endogenous estrogen in postmenopausal women, accompanied by a significant reduction in bone mass [5, 6]. Therefore, estrogens were widely used to treat PMO for a period of time. After hormonal therapy was found to increase the risk of heart disease and breast cancer, the use of estrogens dropped dramatically, even though the risk was low [7]. Several drugs are being used to treat PMO, including bisphosphonates, calcitonin, and other, however, they show adverse effects on the 
gastrointestinal tract [7]. Therefore, it is imperative to understand the pathogenesis of PMO and develop effective therapeutic strategies.

MicroRNAs (miRNAs), as 22-25 nucleotides non-coding small RNAs, play an indispensable role in regulating genes expression in organisms [8]. Emerging evidence shows that miRNAs are implicated in regulating numerous biological processes, such as cell growth, apoptosis, autophagy, differentiation, inflammation, invasion and migration [9-12]. In addition, the function of miRNAs in the development of PMO has attracted a lot of attention. Recent work performed by Liet al. demonstrated that miR-133a participated in the pathogenesis of PMO by facilitating osteoclast differentiation [13]. Moreover, the same group reported that miR-210 was implicated in the development of PMO through increasing vascular endothelial growth factor (VEGF) expression and osteoblast differentiation [14]. Thus, circulating miR-133a-3p might be regarded as an underlying non-invasive biomarker and therapy target in PMO [15]. Several reports documented the involvement of miR-491-3p in cancer, for example, miR-491-3p reduced multidrug resistance of hepatocellular carcinoma, inhibited the growth and invasion of osteosarcoma cells, regulated the chemo-sensitivity of human tongue cancer, and participated in the pathogenesis of clear cell renal cell carcinoma [16-19]. However, its role in $\mathrm{PMO}$ has yet not been investigated.

We found that the expression of miR-491-3p was lower in patients with $\mathrm{PMO}$, while the expression of cathepsin $\mathrm{S}$ (CTSS) in PMO patients showed an opposite trend. Moreover, CTSS was confirmed as a target of miR-491$3 p$. More importantly, we revealed that miR-491-3p could promote the growth and differentiation of hFOB1.19 cells, and inhibit their apoptosis by reducing CTSS expression. Our research provides novel pairs of molecules involved in the pathogenesis of PMO with potential use for the diagnosis and therapy of this syndrome.

\section{Materials and methods}

Data sources. The expression patterns of miR-491-3p and CTSS in PMO were acquired from the GEO database following accession numbers GSE74209 and GSE56116. The GSE74209 dataset including 6 patients with $\mathrm{PMO}$ fractures (osteoporosis) and 6 patients with osteoarthritis ('healthy' control) was used to analyze the expression of miR-491-3p. The GSE56116 dataset containing 10 patients with PMO fractures (osteoporosis) and 3 patients with osteoarthritis ('healthy' control) was utilized to analyze the expression of CTSS.

Cell culture and transfection. Human osteoblast cell line hFOB1.19 was purchased from the Shanghai cell bank of the Chinese Academy of Medical Sciences (Shanghai, China) and cultivated in the $\alpha$-MEM medium including $10 \%$ fetal bovine serum, $100 \mathrm{U} / \mathrm{mL}$ penicillin and $0.1 \mathrm{mg} / \mathrm{mL}$ streptomycin (Invitrogen, Carlsbad, CA, USA). Overexpression of miR-491-3p and CTSS were achieved by transfection of miR-491-3p mimic and pcDNA3.1-CTSS (GenePharma, Shanghai, China) into cells, respectively. The transfection concentrations were $50 \mathrm{nmol} / \mathrm{L}$ for the miR-491-3p mimic, miR-491-3p mimic NC, pcDNA3.1-CTSS and pcDNA3.1. All of them were transfected into hFOB1.19 cells by Lipofectamine 3000 reagent (Invitrogen).

Cell viability test. Cell counting kit-8 (CCK-8, Dojindo Moleular Technologies, Inc., Gathersburg, MD, USA) test was carried out to detect the viability of hFOB1.19 cells. Cells were inoculated in 96-well plates with the density of $1 \times 10^{3}$ cells/well. After cells adhesion, the culture medium was changed, miR-491-3p mimic or miR-491-3p mimic $\mathrm{NC}$ was added, and the cultivation was continued for $48 \mathrm{~h}$. Then, the cells were added with $20 \mu \mathrm{L}$ of CCK- 8 reagent and cultured for another $1.5 \mathrm{~h}$. Finally, microplate reader SpectraMax®iD3 (Molecular Devices, San Jose, CA, USA) was used to measure the optical density (OD) values.

Cell apoptosis test. The apoptosis of treated cells was detected by flow cytometry. Cells were collected and centrifuged. Then, cells were suspended in pre-cooling phosphate buffer saline (PBS) and centrifuged again. Subsequently, the supernatant was sucked out and cells were re-suspended with $1 \times$ binding buffer. We adjusted cell density to $1-5 \times 10^{6}$ cells $/ \mathrm{mL}$. Mixed $5 \mu \mathrm{L}$ of Annexin V/FITC and $100 \mu \mathrm{L}$ of cell suspension into $5 \mathrm{~mL}$ tube, and then the mixture was left for $5 \mathrm{~min}$ in the darkroom. Before detection, $10 \mu \mathrm{L}$ of propidium iodoide (PI) and $400 \mu \mathrm{L}$ of PBS were added into the flow tube. The results were analyzed by Flowjo software 7.6.1.

MiR-491-3p target gene prediction website. The biological prediction websites including MiRanda (http://www.microrna.org), miRWalk (http://zmf.umm.uni-heidelberg.de/apps/ /zmf/mirwalk2/), and TargetScan (http://www.targetscan. org/), miRDB (http://www.mirdb.org/) were used to predict the target genes of miR-491-3p.

Dual luciferase reporter assay. The fragments of CTSS-mutant-type (mut) and CTSS-wild-type (wt) were synthesized and inserted into a pmirGLO vector (GenePharma). The cells were inoculated to 24-well plates. When cells grew to $80 \%$ confluence, luciferase vector containing wt-CTSS or mut-CTSS and miR-491-3p mimic or mimic NC were co-transfected into HEK293 cells by Lipofectamine 3000 reagent (Invitrogen). After $48 \mathrm{~h}$, the protein was extracted and the luciferases activity was detected by Dual-Luciferase Reporter Assay Kit (Promega, Madison, WI, USA) following the manufacturer's specification.

RNA extraction and Quantitative Real-time PCR (qRT-PCR). The whole RNA was obtained from the treated 


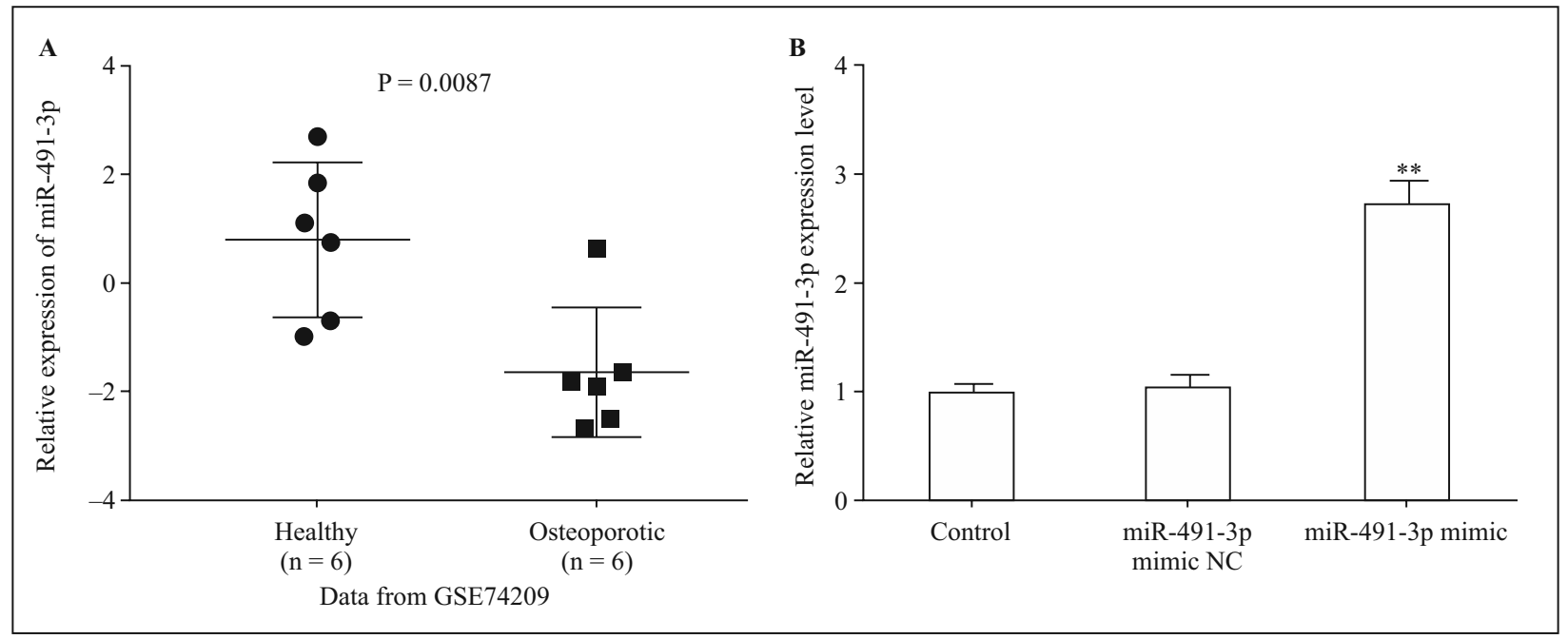

Figure 1. Determination of miR-491-3p expression. A. Data from GEO dataset revealed that miR-491-3p expression was lower in PMO osteoporotic patients $(n=6)$ compared with that of the 'healthy' people $(n=6), P=0.0087$. B. The relative expression of miR-491-3p in hFOB1.19 cells was measured after transfection with miR-491-3p mimic or miR-491-3p mimic $\mathrm{NC},{ }^{* *} \mathrm{P}<0.01$ vs. control.

cells by TRIzol (Invitrogen) and reverse transcribed into cDNA by PrimeScript RT Reagent Kit and Mir- ${ }^{\text {тм }}$ miRNA First Strand Synthesis Kit (Invitrogen). Then, the expression of mRNA and miRNA were analyzed by real-time PCR with the help of SYBR Premix Ex Taq II and SYBR PrimeScriptTM miRNA RT-PCT Kit (TaKaRa, Tokyo, Japan), accordingly. Subsequently, the real-time PCR was performed on a 7900HT real-time PCR system with the following procedures: 40 circles consist of $95^{\circ} \mathrm{C}$ for $5 \mathrm{~min}, 95^{\circ} \mathrm{C}$ for $30 \mathrm{~s}$, then $60^{\circ} \mathrm{C}$ for $45 \mathrm{~s}, 72^{\circ} \mathrm{C}$ for $30 \mathrm{~min}$. GAPDH was considered as the internal standard for mRNA detection, and U6 was regarded as the internal standard for miRNA calculation. $2^{-\Delta \Delta C t}$ method was used to analyze the relative expression of mRNA and miRNA. The sequences of the primers were synthesized as below: MiR-491-3p, forward: 5'-AGTGGGGAACCCTTCC-3', reverse: 5'-GAACATGTCTGCG-TATCTC-3'; U6, forward: 5'-AAAGCAAATCATCGGACGACC-3', reverse: 5'-GTACAACACATTGTTTCCTCGGA-3'; CTSS, forward: 5'-TGGATCACCACT-GGCATCTCTG-3', reverse: 5'-GCTCCAGGTTGTGAAGCATCAC-3'; GAPDH, forward: 5'-TGTGGGCATCAATGGATTTGG-3', reverse: 5'-CCCTCCAGG-GGATCTGTTTG-3'.

Western blotting. Protein samples were extracted from the treated cells by RIPA lysis buffer with protease inhibitor. $20 \mu \mathrm{g}$ of protein within $1 \times$ loading buffer was put into each well, isolated by sodium dodecyl sulphate-polyacrylamide gel electrophoresis and electro-transferred onto PVDF membranes under wet condition. Then $5 \%$ non-fat milk dissolved in TBST (10 mM Tris- $\mathrm{HCl}, \mathrm{pH} 7.5,150 \mathrm{mM} \mathrm{NaCl}$, $0.05 \%$ Tween-20) was used to block the membranes. The membranes were then incubated with primary antibodies against CTSS (1:2000), Bcl-2 (1:1000), Bax (1:2000), Cleaved caspase-3 (1:3000), Cleaved caspase-9 (1:3000), ALP (1:3000), Runx2 (1:1000), Osterix (1:5000), OPN(1:1000) and GAPDH (1:10000) (all from Abcam, Cambridge, UK) at $4^{\circ} \mathrm{C}$ overnight. Next, the membranes were incubated with the IgG-HRP antibody at about $25^{\circ} \mathrm{C}$ for $1 \mathrm{~h}$. Finally, the signals were developed by ECL (HRP Substrate Luminol Reagent: HRP Substrate Peroxide Solution = 1:1) and scanned by QUANTITY ONE software 4.6.6. GAPDH was regarded as the internal standard.

Statistical analysis. All experimental data were repeated at least three times and analyzed by SPSS22.0 and Graphpad Prism 5.0 software. Student's t-test was used to analyze the difference between two samples, while the comparison among multiple groups was calculated by ANOVA followed by Dunnett (compare all groups $v s$ control group) and Bofferornie (compare selected pairs of groups) post hoc test. $\mathrm{P}$ value less than 0.05 was considered to indicate a statistically significant difference.

\section{Results}

\section{The expression of MiR-491-3p was lower} in patients with $P M O$

Firstly, GEO dataset was used to detect the expression of miR-491-3p in PMO. The data in Figure 1A manifested that miR-491-3p expression was lower in patients with PMO fracture (osteoporotic, $\mathrm{n}=6$ ) than that of the patients with osteoarthritis ("healthy" control, $\mathrm{n}=6, \mathrm{P}=0.0087$ ). Subsequently, to inves- 


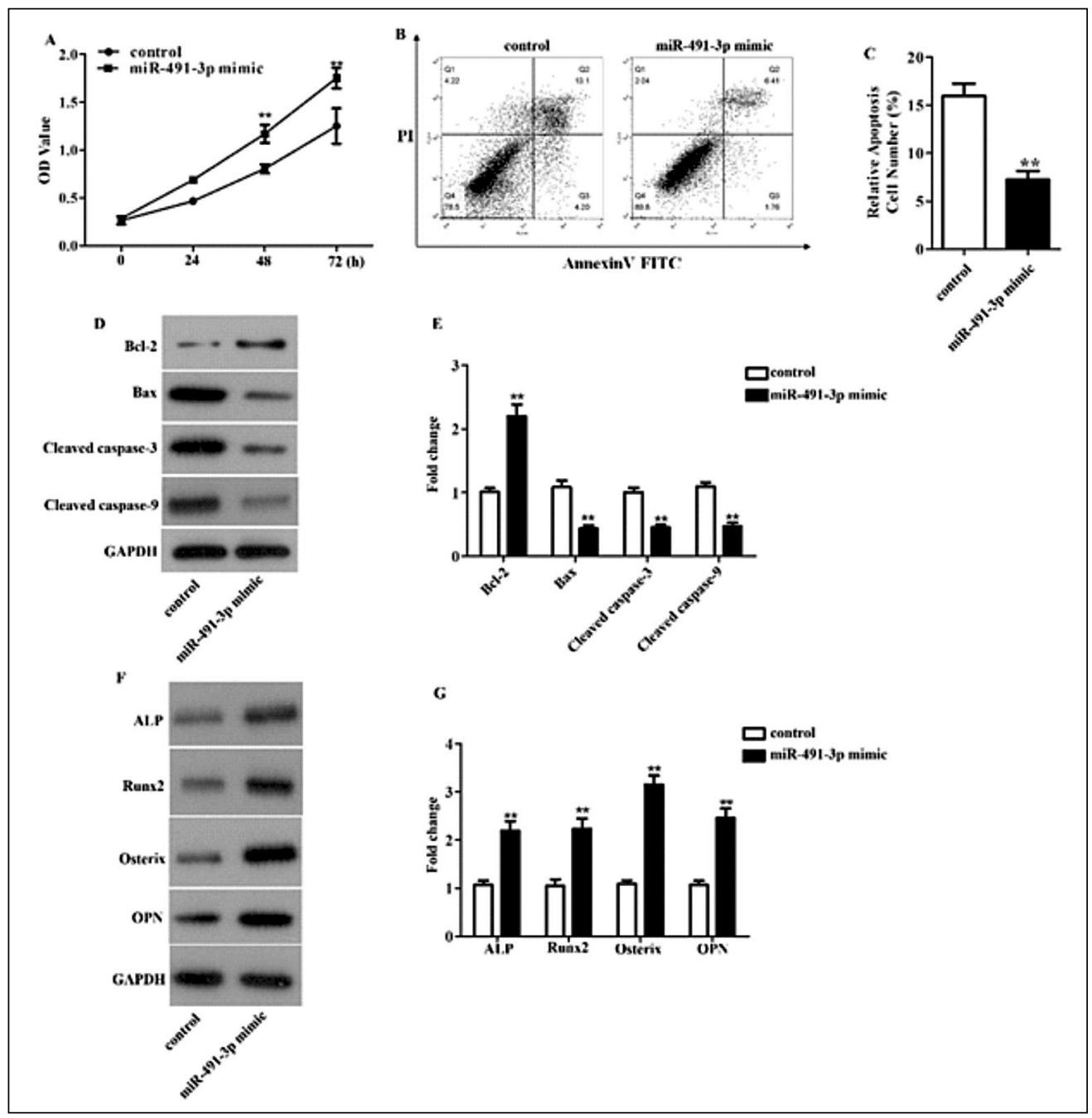

Figure 2. MiR-491-3p promoted proliferation and differentiation of hFOB1.19 cells, and inhibited their apoptosis. A. The proliferation of hFOB1.19 cells was measured by CCK-8 assay as described in Methods. B-C. The apoptosis of hFOB1.19 cells was detected by flow cytometry. D-E. The a presence of poptosis-related proteins in hFOB1.19 cells were analyzed by Western blotting. F-G. The presence of differentiation-related proteins in hFOB1.19 cells were measured by Western blotting. ${ }^{* *} \mathrm{P}<0.01$ vs. control.

tigate the effects of miR-491-3p on hFOB1.19 cells, we attempted to up-regulate miR-491-3p with mimic. The data presented in Figure 1B indicated that miR491-3p expression was significantly upregulated in hFOB1.19 cells after treatment with miR-491-3p mimic compared with that of the control cells and miR-491-3p mimic $\mathrm{NC}$ groups $(\mathrm{P}<0.01)$.

\section{Overexpression of miR-491-3p could inhibit the apoptosis of hFOB1.19 cells and promote their proliferation and differentiation}

To further explore the impact of miR-491-3p on hFOB1.19 cells proliferation, apoptosis and differentiation, CCK-8, flow cytometry and western blotting assays were carried out. The data of CCK-8 tests showed that up-regulation of miR-491-3p could increase the OD values of hFOB1.19 cells compared with that of the control and the difference was significant at $48 \mathrm{~h}$ and $72 \mathrm{~h}$ (Fig. 2A, $\mathrm{P}<0.01$ ).

Flow cytometry results demonstrated that the apoptosis rate of hFOB1.19 cells was reduced nearly $60 \%$ after upregulation of miR-491-3p compared with that of the control (Figs. 2B-C, P $<0.01$ ). Subsequently, the apoptotic-related proteins were analyzed by Western blotting. The results indicated that the expression of anti-apoptotic protein Bcl-2 was reduced, and the expression of pro-apoptotic proteins including Bax, Cleaved caspase- 3 and Cleaved caspase- 9 were increased in hFOB1.19 cells after transfection of the cells with miR-491-3p mimic (Figs. 2D-E, P < 0.01).

To study the effects of miR-491-3p on hFOB1.19 cells differentiation, we analyzed the changes of differentiation-related proteins with western blotting. The results indicated that after miR-491-3p mimic 


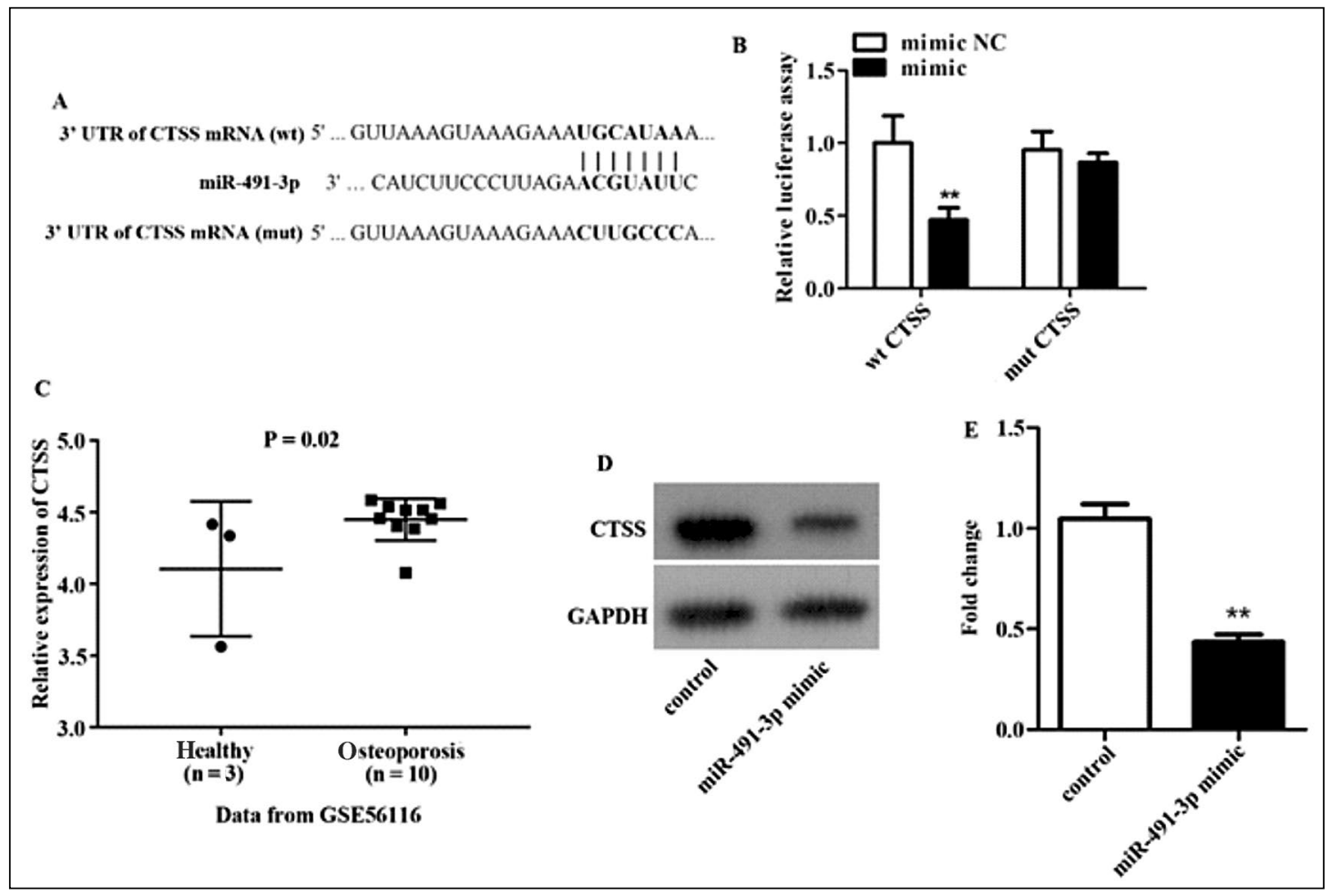

Figure 3. CTSS was highly expressed in PMO and negatively regulated by miR-491-3p. A. The sequences of wild type 3'UTR of CTSS, mutant type 3'UTR of CTSS, and miR-491-3p were presented. B. The luciferase activity of wt CTSS and mut CTSS were assessed after the cells were transfected with miR-491-3p mimic or NC. C. The data from GEO indicated that CTSS was clearly up-regulated in patients with PMO $(n=10)$ compared with that of the 'healthy' people $(n=3), P=0.02$. D-E. The expression of CTSS was detected after transfected with miR-491-3p mimic or NC. **P $<0.01$ vs. control.

treatment, the expression of ALP, Runx2, Osterix and OPN significantly increased to $2.05,2.13,2.9$, and 2.3 times of the control group, respectively (Figs. 2F-G, P $<0.01$ ). Thus, the results of all performed tests indicated that up-regulation of miR-491-3p could increase the proliferation and differentiation of hFOB1.19 cells, and reduce the apoptosis of hFOB1.19 cells.

\section{Cathepsin $S$ was highly expressed in patients with $P M O$ and negatively regulated by $\mathrm{miR}-491-3 p$}

Based on the results from target gene prediction website and bioinformatics analysis, CTSS was selected as a target of miR-491-3p for further investigation. As presented in Figure 3A, the wt 3'UTR of CTSS contains the complementary binding sequence of miR-491-3p. Subsequently, dual luciferase reporter assay was performed to confirm the association between miR491-3p and CTSS. The data from Figure 3B showed that the luciferase activity in wt 3'UTR of CTSS was clearly decreased after transfected with miR-491-3p mimic compared with that of the miR-491-3p mimic NC group $(\mathrm{P}<0.01)$. However, miR-491-3p had little effect on luciferase activity of the mut 3'UTR of CTSS. The results further confirmed that miR-491-3p directly binds with CTSS in osteoblastic cell line. The data from GEO database indicated that CTSS was significantly up-regulated in patients with PMO fracture (osteoporotic, $\mathrm{n}=10$ ) compared with that of the patients in osteoarthritis ("healthy" control, $\mathrm{n}=3, \mathrm{P}=0.02$, Fig. $3 \mathrm{C}$ ). Moreover, the expression of CTSS was clearly reduced in hFOB1.19 cells after transfected with miR-491-3p mimic (Figs. 3D-E, $\mathrm{P}<0.01)$. The results implied that CTSS was a direct target of miR-491-3p and was negatively modulated by miR-491-3p.

\section{CTSS suppressed the positive effects of miR-491-3p on hFOB1.19 cells}

To explore the relationships between miR-491-3p and CTSS in PMO, the biological properties of hFOB1.19 cells were analyzed after they were treated with miR491-3p mimic and pcDNA3.1-CTSS. The results shown in Figure 4A indicated that the OD values of hFOB1.19 cells were increased after treatment with miR-491-3p mimic in contrast with that of the control, while the raising tendency was reversed in miR-491-3p mimic and pcDNA3.1-CTSS group $(\mathrm{P}<0.01)$. The data indicated that CTSS could suppress the ascend- 


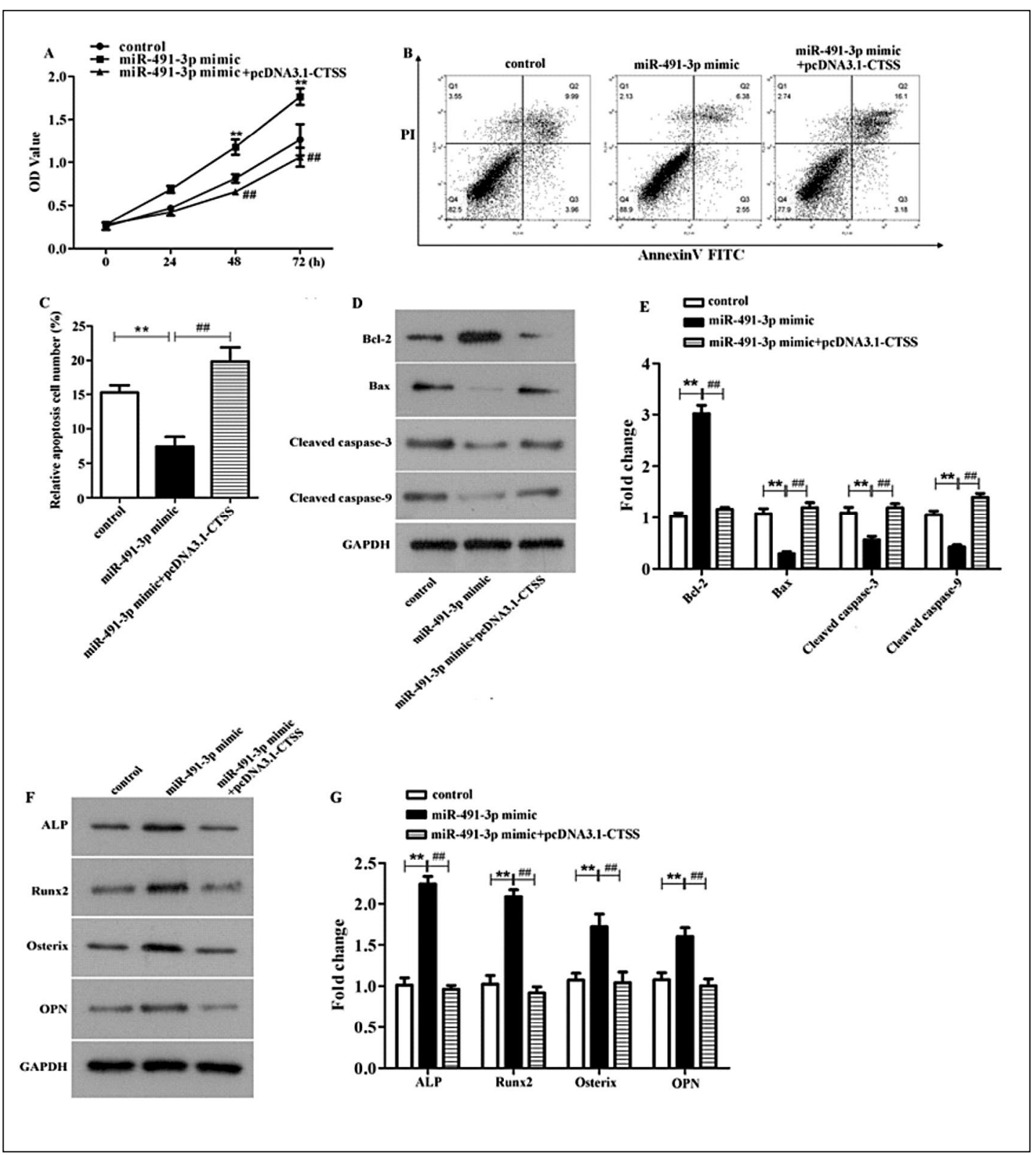

Figure 4. CTSS suppressed the promoting effects of miR-491-3p on the proliferation and differentiation of hFOB1.19 cells, and the inhibitory effects of miR-491-3p on hFOB1.19 cells apoptosis. A. The hFOB1.19 cells proliferation was detected by CCK-8 after their treatment with miR-491-3p mimic/miR-491-3p mimic + pcDNA3.1-CTSS. B-C. Flow cytometry was applied to detect the apoptosis of hFOB1.19 cells. D-E. The apoptosis-related proteins were measured by Western blotting. F-G. The expression of ALP, Runx2, Osterix and OPN were detected by Western blotting. ${ }^{* *} \mathrm{P}<0.01,{ }^{\# \#} \mathrm{P}<0.01$, ${ }^{* *}$ represented miR-491-3p mimic $v s$ control, \#\# represented miR-491-3p mimic and pcDNA3.1-CTSS $v s$ miR-491-3p mimic.

ing proliferation ability of hFOB1.19 cells induced by miR-491-3p mimic. Flow cytometry data showed that the apoptosis of hFOB1.19 cells was reduced nearly 50\% after treatment with miR-491-3p mimic compared with that of the control, and increased approximately two times after treatment with miR491-3p mimic and pcDNA3.1-CTSS compared with that of the miR-491-3p mimic group (Figs. 4B-C, $\mathrm{P}<0.01)$. Moreover, the expression of anti-apoptotic protein Bcl-2 was reduced about $60 \%$, and the pro-apoptotic proteins Bax, Cleaved caspase- 3 and Cleaved caspase- 9 were significantly increased after the cells were transfected with miR-491-3p mimic and pcDNA3.1-CTSS compared with that of the miR-491-3p mimic group $(\mathrm{P}<0.01)$. On the contrary, the raising tendency of ALP, Runx2, Osterix and OPN expression induced by miR-491-3p mimic was reversed after the cells were transfected with miR-491-3p mimic and 
pcDNA3.1-CTSS $(\mathrm{P}<0.01)$. All the data implicate that CTSS could suppress the promoting effects of miR-491-3p on hFOB1.19 cells proliferation and differentiation, and repress the inhibitory effects of miR-491-3p on hFOB1.19 cells apoptosis.

\section{Discussion}

$\mathrm{PMO}$ is an insidious disease without any obvious symptoms in its early stage. Once the signs of PMO appear, the fracture has already occurred [20]. Therefore, it is essential to search effective and preventive measures and molecular markers for early diagnosis. With the development of more and more validated miRNA signatures and mature medium-throughput methods in the clinical setting, specific miRNA markers are raised potentially to conduce to human health [21]. Recently, several researchers have discovered that some miRNAs including miR-491-3p were modulated by enterovirus 71 which, apart from other virus types, may cause of major outbreaks of hand, foot, and mouth disease (HFMD) [22]. MiR-491-3p has been reported to be involved in the pathogenesis of major depression or suicide [23]. In addition, previous study has found that miR-491-3p played a vital role in the modulation of multidrug resistance in hepatocellular carcinoma through regulating ABCB1 and Sp3 expression [19]. Moreover, miR491-3p was discovered to suppress the growth and invasiveness of osteosarcoma [18] and glioblastoma [24] cells. However, study of miR-491-3p in osteoporosis has yet not been reported. In the current study, the data indicated that miR-491-3p expression was lower in PMO patients. Importantly, there are reports which indicate that proliferation and differentiation of osteoblasts are important for the development of osteoporosis [25]. ALP, Runx2, Osterix and OPN have been demonstrated to play positive roles in promoting osteogenesis [26]. In our study, the upregulation of miR-491-3p increased the expression of ALP, Runx2, Osterix and OPN, indicating that miR-491-3p can promote osteoblast differentiation. Cell apoptosis is an important biological process that occurs in many diseases, including PMO [27]. In our study, up-regulation of miR-491-3p attenuated the apoptosis of human osteoblastic cells. These results suggested that miR-491-3p inhibited the development of PMO by promoting the growth and differentiation of osteoblasts, and suppressing their apoptosis.

It is well known that miRNAs perform their functions through binding to mRNAs and blocking protein expression. Based on the biological predictions, bioinformatical analysis and dual luciferase reporter assay, cathepsin $\mathrm{S}$ was selected and confirmed as a direct target of miR-491-3p. CTSS, as a lysosomal protease, can promote the degradation of damaged or unnecessary proteins in the lysosomal pathway [28], and was reported to be involved in several diseases including atherosclerosis, tumor metastasis and osteoporosis [29]. In addition, growing evidence have indicated that CTSS regulated adipocyte and osteoblast differentiation, bone turnover, and bone microarchitecture [30]. Furthermore, CTSS was also used as a potential drug target for osteoporosis and rheumatoid arthritis [31]. In our study, CTSS was verified as a direct target of miR-491-3p, and was negatively modulated by miR-491-3p. Moreover, data from GEO dataset showed that CTSS was highly expressed in patients with PMO. Rescue assays indicated that overexpression of CTSS could limit the positive effects of miR-491-3p on osteoblastic cells. More in vivo experiments are needed to confirm these results, since in vitro experiments cannot fully simulate the situation in vivo.

In conclusion, our findings discovered that miR491-3p showed a tendency of low expression in patients with PMO, while the expression trend of CTSS in PMO was just the opposite. What's more, overexpression of miR-491-3p could promote osteoblasts' proliferation and differentiation, and inhibit their apoptosis. In addition, CTSS was verified as a target gene of miR-491-3p and was found to be negatively regulated by miR-491-3p in osteoblastic cells. Further experiments implied that overexpression of CTSS could suppress the promoting effects of miR-491-3p on the phenotype of hFOB1.19 cells. To sum up, miR491-3p could promote the differentiation and maintenance of osteoblasts by reducing CTSS expression. The results of this study provide a pair of molecules for the potential diagnosis and treatment of PMO.

\section{References}

1. Shao M. Construction of an miRNA-Regulated Pathway Network Reveals Candidate Biomarkers for Postmenopausal Osteoporosis. Comput Math Methods Med. 2017; 2017: 9426280, doi: 10.1155/2017/9426280, indexed in Pubmed: 29158773.

2. Bliuc D, Alarkawi D, Nguyen TV, et al. Risk of subsequent fractures and mortality in elderly women and men with fragility fractures with and without osteoporotic bone density: the Dubbo Osteoporosis Epidemiology Study. J Bone Miner Res. 2015; 30(4): 637-646, doi: 10.1002/jbmr.2393, indexed in Pubmed: 25359586.

3. Bliuc D, Nguyen ND, Nguyen TV, et al. Compound risk of high mortality following osteoporotic fracture and refracture in elderly women and men. J Bone Miner Res. 2013; 28(11): 2317-2324, doi: 10.1002/jbmr.1968, indexed in Pubmed: 23616397.

4. Jilka RL, O'Brien CA. The Role of Osteocytes in Age-Related Bone Loss. Curr Osteoporos Rep. 2016; 14(1): 16-25, doi: 10.1007/s11914-016-0297-0, indexed in Pubmed: 26909563.

5. Marcus R. Post-menopausal osteoporosis. Best Pract Res Clin Obstet Gynaecol. 2002; 16(3): 309-327, doi: 10.1053/ beog.2002.0284, indexed in Pubmed: 12099665. 
6. Seibel MJ, Dunstan CR, Zhou H, et al. Sex steroids, not FSH, influence bone mass. Cell. 2006; 127(6): 1079; author reply 1080-1079; author reply 1081, doi: 10.1016/j.cell.2006.12.002, indexed in Pubmed: 17174881.

7. Tella SH, Gallagher JC. Prevention and treatment of postmenopausal osteoporosis. J Steroid Biochem Mol Biol. 2014; 142: 155-170, doi: 10.1016/j.jsbmb.2013.09.008, indexed in Pubmed: 24176761.

8. He L, Hannon GJ. MicroRNAs: small RNAs with a big role in gene regulation. Nat Rev Genet. 2004; 5(7): 522-531, doi: 10.1038/nrg1379, indexed in Pubmed: 15211354.

9. Stefani G, Slack FJ. Small non-coding RNAs in animal development. Nat Rev Mol Cell Biol. 2008; 9(3): 219-230, doi: 10.1038/nrm2347, indexed in Pubmed: 18270516.

10. Filipowicz W, Bhattacharyya SN, Sonenberg N. Mechanisms of post-transcriptional regulation by microRNAs: are the answers in sight? Nat Rev Genet. 2008; 9(2): 102-114, doi: 10.1038/nrg2290, indexed in Pubmed: 18197166.

11. Olejniczak M, Kotowska-Zimmer A, Krzyzosiak W. Stress-induced changes in miRNA biogenesis and functioning. Cell Mol Life Sci. 2018; 75(2): 177-191, doi: 10.1007/s00018-0172591-0, indexed in Pubmed: 28717872.

12. Yu A, Zhang T, Zhong W, et al. miRNA-144 induces microglial autophagy and inflammation following intracerebral hemorrhage. Immunol Lett. 2017; 182: 18-23, doi: 10.1016/j. imlet.2017.01.002, indexed in Pubmed: 28062218.

13. Li Z, Zhang W, Huang Y. MiRNA-133a is involved in the regulation of postmenopausal osteoporosis through promoting osteoclast differentiation. Acta Biochim Biophys Sin (Shanghai). 2018; 50(3): 273-280, doi: 10.1093/abbs/gmy006, indexed in Pubmed: 29425279.

14. Liu XD, Cai F, Liu L, et al. MicroRNA-210 is involved in the regulation of postmenopausal osteoporosis through promotion of VEGF expression and osteoblast differentiation. Biol Chem. 2015; 396(4): 339-347, doi: 10.1515/hsz-2014-0268, indexed in Pubmed: 25503465.

15. Pala E, Denkçeken T. Differentially expressed circulating miRNAs in postmenopausal osteoporosis: a meta-analysis. Biosci Rep. 2019; 39(5), doi: 10.1042/BSR20190667, indexed in Pubmed: 31023966.

16. He H, Wang L, Zhou W, et al. MicroRNA Expression Profiling in Clear Cell Renal Cell Carcinoma: Identification and Functional Validation of Key miRNAs. PLoS One. 2015; 10(5): e0125672, doi: 10.1371/journal.pone.0125672, indexed in Pubmed: 25938468.

17. Zheng G, Jia X, Peng C, et al. The miR-491-3p/mTORC2/ FOXO1 regulatory loop modulates chemo-sensitivity in human tongue cancer. Oncotarget. 2015; 6(9): 6931-6943, doi: 10.18632/oncotarget.3165, indexed in Pubmed: 25749387.

18. Duan J, Liu J, Liu Y, et al. miR-491-3p suppresses the growth and invasion of osteosarcoma cells by targeting TSPAN1. Mol Med Rep. 2017; 16(4): 5568-5574, doi: 10.3892/ mmr.2017.7256, indexed in Pubmed: 28849017.

19. Zhao Y, Qi X, Chen J, et al. The miR-491-3p/Sp3/ABCB1 axis attenuates multidrug resistance of hepatocellular carci- noma. Cancer Lett. 2017; 408: 102-111, doi: 10.1016/j.canlet.2017.08.027, indexed in Pubmed: 28844709.

20. Lippuner K. Medical treatment of vertebral osteoporosis. Eur Spine J. 2003; 12 Suppl 2: S132-S141, doi: 10.1007/s00586003-0608-x, indexed in Pubmed: 13680313.

21. Backes C, Meese E, Keller A. Specific miRNA disease biomarkers in blood, serum and plasma: challenges and prospects. Mol Diagn Ther. 2016; 20(6): 509-518, doi: 10.1007/ s40291-016-0221-4, indexed in Pubmed: 27378479.

22. Bian L, Wang Y, Liu Q, et al. Prediction of signaling pathways involved in enterovirus 71 infection by algorithm analysis based on miRNA profiles and their target genes. Arch Virol. 2015; 160(1): 173-182, doi: 10.1007/s00705-014-2249-2, indexed in Pubmed: 25287131.

23. Serafini G, Pompili M, Hansen KF, et al. The involvement of microRNAs in major depression, suicidal behavior, and related disorders: a focus on miR-185 and miR-491-3p. Cell Mol Neurobiol. 2014; 34(1): 17-30, doi: 10.1007/s10571-0139997-5, indexed in Pubmed: 24213247.

24. Li X, Liu Y, Granberg KJ, et al. Two mature products of MIR-491 coordinate to suppress key cancer hallmarks in glioblastoma. Oncogene. 2015; 34(13): 1619-1628, doi: 10.1038/ onc.2014.98, indexed in Pubmed: 24747968.

25. Fan JZ, Yang L, Meng GL, et al. Estrogen improves the proliferation and differentiation of hBMSCs derived from postmenopausal osteoporosis through notch signaling pathway. Mol Cell Biochem. 2014; 392(1-2): 85-93, doi: 10.1007/ s11010-014-2021-7, indexed in Pubmed: 24752351.

26. An J, Yang H, Zhang Q, et al. Natural products for treatment of osteoporosis: The effects and mechanisms on promoting osteoblast-mediated bone formation. Life Sci. 2016; 147 : 46-58, doi: 10.1016/j.lfs.2016.01.024, indexed in Pubmed: 26796578.

27. Yan L, Guo N, Cao Y, et al. miRNA145 inhibits myocardial infarctioninduced apoptosis through autophagy via Akt3/ mTOR signaling pathway in vitro and in vivo. Int J Mol Med. 2018; 42(3): 1537-1547, doi: 10.3892/ijmm.2018.3748, indexed in Pubmed: 29956747.

28. Wilkinson RDA, Williams R, Scott CJ, et al. Cathepsin S: therapeutic, diagnostic, and prognostic potential. Biol Chem. 2015; 396(8): 867-882, doi: 10.1515/hsz-2015-0114, indexed in Pubmed: 25872877.

29. Wilder CL, Park KY, Keegan PM, et al. Manipulating substrate and $\mathrm{pH}$ in zymography protocols selectively distinguishes cathepsins $\mathrm{K}, \mathrm{L}, \mathrm{S}$, and $\mathrm{V}$ activity in cells and tissues. Arch Biochem Biophys. 2011; 516(1): 52-57, doi: 10.1016/j. abb.2011.09.009, indexed in Pubmed: 21982919.

30. Rauner M, Föger-Samwald U, Kurz MF, et al. Cathepsin $\mathrm{S}$ controls adipocytic and osteoblastic differentiation, bone turnover, and bone microarchitecture. Bone. 2014; 64: 281-287, doi: 10.1016/j.bone.2014.04.022, indexed in Pubmed: 24780878

31. Yasuda Y, Kaleta J, Brömme D. The role of cathepsins in osteoporosis and arthritis: rationale for the design of new therapeutics. Adv Drug Deliv Rev. 2005; 57(7): 973-993, doi: 10.1016/j.addr.2004.12.013, indexed in Pubmed: 15876399.

Submitted: 23 September, 2019

Accepted after reviews: 6 February, 2020 Available as AoP: 16 March, 2020 